\title{
Dynamic patterns of mechanical stimulation co-localise with growth and cell proliferation during morphogenesis in the avian embryonic knee joint
}

\author{
Karen A. Roddy ${ }^{\mathrm{a}, \mathrm{b}}$, Geraldine M. Kelly ${ }^{\mathrm{c}}$, Maarten H. van Es ${ }^{\mathrm{c}}$, Paula Murphy ${ }^{\mathrm{a}, \mathrm{b}}$, Patrick J. Prendergast ${ }^{\mathrm{a}, *}$ \\ a Trinity Centre for Bioengineering, School of Engineering, Trinity College Dublin, Ireland \\ ${ }^{\mathrm{b}}$ Department of Zoology, School of Natural Sciences, Trinity College Dublin, Ireland \\ ${ }^{c}$ Nanoscale Function Group, Conway Institute of Biomolecular and Biomedical Research, University College Dublin, Ireland
}

\section{A R T I C L E I N F O}

Article history:

Accepted 31 August 2010

Keywords:

Mechanobiology

Joint development

Finite element analysis

Articular cartilage development

Muscle contraction

\begin{abstract}
A B S T R A C T
Muscle contractions begin in early embryonic life, generating forces that regulate the correct formation of the skeleton. In this paper we test the hypothesis that the biophysical stimulation generated by muscle forces may be a causative factor for the changes in shape of the knee joint as it grows. We do this by predicting the spatial and temporal patterns of biophysical stimuli, where cell proliferation and rudiment shape changes occur within the emerging tissues of the joint over time. We used optical projection tomography (OPT) to create anatomically accurate finite element models of the embryonic knee at three time points (stages) of development. OPT was also used to locate muscle attachment sites and AFM was used to determine material properties. An association was found between the emergence of joint shape, cell proliferation and the pattern of biophysical stimuli generated by embryonic muscle contractions. Elevated rates of growth and cell proliferation in the medial condyle were found to co-localise with elevated patterns of biophysical stimuli including maximum principal stresses and fluid flow, throughout the time period studied, indicating that cartilage growth and chondrocyte proliferation in the epiphysis is potentially related to local patterns of biophysical stimuli. The development of the patella and articular cartilages, which is known to be affected by in ovo immobilisation, could be contributed to by specific patterns of fluid flow, pore pressure and stress in the joint interzone. This suggests that both cartilage growth and tissue differentiation in the embryonic joint is regulated by specific patterns of biophysical stimuli and that these stimuli are needed for the correct development of the joint.
\end{abstract}

(c) 2010 Elsevier Ltd. All rights reserved.

\section{Introduction}

Synovial joints have intricate 3D shapes, which are highly adapted for their biomechanical function. They emerge through the division of mesenchymal condensations, forming cartilage templates interrupted by an interzone, a dense avascular region from which all the tissues of the joint are derived (Koyama et al., 2008). Shape emerges within the cartilage template as the chondrocytes proliferate and secrete matrix, causing them to grow until they undergo hypertrophy leading to ossification. Within this system a variety of joint shapes are created through some unknown modulation of regulatory mechanisms (Wilsman et al., 1996).

Mechanical forces have been implicated in the process of joint morphogenesis. Paralysis was reported to cause flattening and distortion of the articular surfaces (Drachman and Sokoloff, 1966),

\footnotetext{
* Corresponding author.

E-mail address: pprender@tcd.ie (P.J. Prendergast).

URL: http://www.tcd.ie/bioengineering (P.J. Prendergast).
}

reduction in the volume of epiphyseal cartilage (Osborne et al., 2002), the loss of functional outgrowths (Murray and Drachman, 1969; Persson, 1983) and reduction or loss of joint-associated structures such as the patella and articular cartilage (Mikic et al., 2000; Osborne et al., 2002). Cell proliferation rates in the developing growth plate and chondroepiphysis were also downregulated by the removal of embryonic muscle contraction (Germiller and Goldstein, 1997).

Previous studies used computational simulations to predict mechanical forces in simplified representations of the developing skeleton. Heegaard et al. (1999) simulated the emergence of a basic interlocking joint morphology in response to mechanically stimulated differential growth. Other models have been implemented to investigate various aspects of limb development including endochondral ossification and the development of secondary sites of ossification (Carter and Wong, 1988; Stevens et al., 1999), the emergence of the femoral bicondylar angle, and the common childhood orthopaedic problem, developmental dysplasia of the hip (Shefelbine and Carter, 2004; Shefelbine et al., 2002). Nowlan et al. (2008a) created a Finite Element (FE) model from morphologically accurate 3D data captured from the developing chick 
tibiotarsus that was used to investigate the mechanoregulation of ossification by the dynamic patterns of stimuli generated during muscle contraction. A correlation between the patterns of stimuli and the expression patterns of a number of genes involved in bone formation was also found (Nowlan et al., 2008b).

Tanck et al. (2004) analysed late gestation mouse rib rudiments using four point bending tests while Mikic et al. (2004) performed stress-relaxation tests on cores of late stage chick embryonic cartilage, in order to characterise Young's modulus of the cartilage. No studies of the mechanical properties of the interzone were found in the literature by these authors. Atomic force microscopy (AFM) has previously been used as a type of nanoindenter in order to analyse the mechanical properties of biological specimens including growth plate cartilage (Allen and Mao, 2004; Radhakrishnan et al., 2004), articular cartilage (Stolz et al., 2004) and single cells (Rotsch et al., 1997).

In this paper we seek to test the hypothesis that during joint development local changes in cartilage growth and cell proliferation are related to distinct patterns of biophysical stimuli generated by embryonic muscle contraction. To test this hypothesis we used Optical Projection Tomography (OPT) to create an FE simulation, to determine whether dynamic patterns of biophysical stimuli co-localise with cartilage growth and cell proliferation. Embryonic stages from (Hamburger and Hamilton (HH) (1951) stage HH30 to HH34 were chosen as they represent the time period covering the emergence of complex joint shape and the initiation of joint cavity formation (Roddy et al., 2009). New data on the mechanical properties of the embryonic cartilage and interzone tissue were needed, and these were obtained using AFM. Correspondence between patterns of biophysical stimuli, tissue growth and cell proliferation were explored to establish whether dynamic biophysical stimuli co-localise with morphogenetic events. Such a finding would suggest that the patterns of biophysical stimuli distributed in the skeleton act as a type of positional information differentially up- or down-regulating growth, leading to the emergence of specific skeletal shapes.

\section{Method}

\subsection{Finite element analysis}

\subsubsection{Geometry}

The FE model was built from a 3D dataset described in Roddy et al. (2009) consisting of 3D representations of the developing cartilage, tendon, muscle and joint capsule, captured using a range of markers and scanned using Optical Projection Tomography (OPT). 3D images in VTK (Visualisation Toolkit) format, representing the cartilaginous elements of the knee, were extracted from OPT scans of alcian blue stained limbs at (Hamburger and Hamilton, 1951) stages, HH30, HH32 and HH34 (Fig. 1a), and converted into a format suitable for Abaqus FE software, as per Nowlan et al. (2008a). A fourth mesh was generated that encapsulated the interzone and represented the area separating the three skeletal elements, bounded dorsally by the joint capsule and ventrally by muscle masses. Two models representative of each stage were built, as in example Fig. 1b.

\subsubsection{Loading and boundary conditions}

The 3D tendon and muscle data of the developing chick knee joint were examined, and the major dorsal (D1, D2) and ventral (V1, V2) muscles of the thigh were identified (Fig. 1c). The transverse cross-sectional area of each muscle was taken at the longitudinal midpoint of the muscle mass (Fig. 1d), and the resultant forces were calculated using a force per unit area value for embryonic chick muscle of $1.11 \mathrm{mN} / \mathrm{mm}$, derived from Landmesser and Morris (1975) $(n=2$, Table 1). The attachment points for each muscle were located on the rudiment using a combination of visual comparison of external views and virtual sectioning through the $3 \mathrm{D}$ representations of the cartilage, muscle and tendon data at corresponding stages.

As per Tanck et al. (2000) and Nowlan et al. (2008a), a loading cycle consisted of two muscle twitches, a flexion (Fig. 1e) followed by an extension (Fig. 1f) contraction lasting for $1.4 \mathrm{~s}$ and including a ramp-up, hold and ramp-down. A poroelastic analysis was performed using the published values for cartilage permeability $(6.7 \times 10 \mathrm{~m} / \mathrm{Ns})$ and Poisson's ratio $(0.25)$ (Tanck et al., 2000).
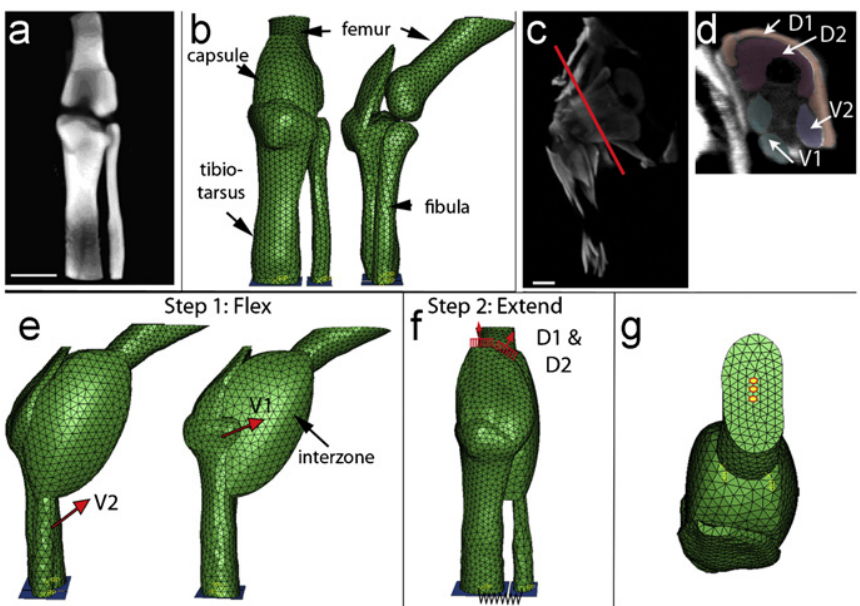

Fig. 1. . 3D data from optical projection tomography (OPT) imaging of alcian blue stained tissue (a) was used to create FE meshes (b) of the developing knee joint (stage HH34 shown) capsule data from ColXI expression (not shown). The magnitude of muscle loads were derived from anti-myosin stained limbs (c) by measuring the cross-sectional areas of each muscle (d); D1, D2, dorsal masses; V1, $\mathrm{V} 2$, ventral masses. The location of the cross-section is indicated by the red line (c). The placement of both the flexion (e, V1, V2) and extension (f, D1, D2) loads was derived from anti-tenascin and scleraxis OPT data. In the model three nodes on the proximal surface are fixed in all three directions $(\mathrm{g})$. Scale bar indicates $1 \mathrm{~mm}$.

A porosity of 0.8 was used (Kelly and Prendergast, 2005). The permeability of the interzone was considered as equivalent to cartilage (a parametric study of permeability in the interzone ranging from $\pm 25 \%$ demonstrated that altering permeability did not alter the resulting pattern of stimuli significantly).

The four mesh elements were connected using tie constraints to represent the connections between skeletal elements and the interzone. Three nodes on the proximal surface of the femoral mesh were fully restrained, while the rest of the nodes on this surface were restrained in the vertical direction only (Fig. 1g). Two rigid plates, attached to the distal surfaces of the tibiotarsus and fibula meshes (Fig. 1f), were connected by a spring to simulate the presence of tissue separating the two cartilaginous elements (Fig. 1f). The spring stiffness is given in Table 2. A zero pore pressure boundary condition was specified on all the external nodes and on the various master surfaces. For each simulation of a flexion/ extension cycle the following parameters were determined: maximum and minimum principal stress and strain, the Von Mises stress, relative fluid-solid velocity and hydrostatic pressure.

\subsubsection{Material properties}

AFM was used to determine Young's modulus of embryonic cartilage and interzone at stage $\mathrm{HH} 32$. Unfixed tissue was equilibrated in $30 \%$ sucrose and frozen in Tissue-Tek embedding compound, cryo-sectioned longitudinally (60 $\mu \mathrm{m}$; Bright 5040) and mounted onto polylysin coated slides (Menzel Glaser).

The AFM was a JPK Nanowizard model mounted onto a Nicon Ti/E microscope. Prior to analysis the beam shaped cantilevers mounted with $5 \mu \mathrm{m}$ borosilicate spheres (Novascan), with a nominal spring constant of $0.95 \mathrm{~N} / \mathrm{m}$, were calibrated. The sensitivity was calibrated by performing a force curve on glass. The spring constant was calibrated using the thermal method (described by Hutter and Bechhoefer, 1993). The tip was then positioned over the area of interest and a force map of $10000 \mathrm{um}^{2}$ was generated with a total of 100 force curves; the map was occasionally reduced to $5000 \mu^{2}$. Samples were indented at approximately $0.75 \mu \mathrm{m} / \mathrm{s}$. Cartilage and interzone tissues were indented with a force of approximately 10 and $4 \mathrm{nN}$, respectively. At least two independent force maps per tissue type were performed $(n=4)$.

Young's moduli for both cartilage and interzone at HH32 were determined by fitting the Hertz model (Hertz, 1882; Sneddon, 1965) to the force curves. The resulting Young's moduli were $0.535 \pm 0.072$ and $0.287 \pm 0.066 \mathrm{kPa}$ for embryonic cartilage and interzone tissues, respectively $(n=4)$.

\subsection{Analysis of changes over time}

\subsubsection{Analysis of developing joint shape}

To capture one aspect of the growth profile of the distal femur, virtual sections captured in a consistent plane through the femoral condyles were prepared from alcian blue stained, OPT scanned limbs presented by Roddy et al. (2009) at stages HH30, HH32 and HH34 $(n=4)$. Since OPT reconstructions produce 3D digital representations, section planes can be varied in all orientations and assessed from multiple views (Summerhurst et al., 2008) allowing distinct morphological 
Table 1

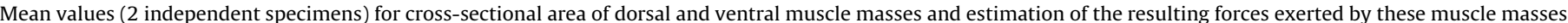
during contraction across developmental stages HH30-34.

\begin{tabular}{|c|c|c|c|c|c|c|}
\hline \multirow[t]{2}{*}{ Specimen number } & \multicolumn{2}{|l|}{ НH30 } & \multicolumn{2}{|l|}{ HH32 } & \multicolumn{2}{|l|}{ HH34 } \\
\hline & Area $\left(\mathrm{mm}^{2}\right)$ & Force $(\mathrm{mN})$ & Area $\left(\mathrm{mm}^{2}\right)$ & Force $(\mathrm{mN})$ & Area $\left(\mathrm{mm}^{2}\right)$ & Force $(\mathrm{mN})$ \\
\hline \multicolumn{7}{|l|}{ Dorsal } \\
\hline 1 & $0.52 \pm 0.02$ & 0.579 & $0.81 \pm 0.14$ & 0.896 & $0.86 \pm 0.04$ & 0.949 \\
\hline 2 & $0.51 \pm 0.03$ & 0.565 & $0.74 \pm 0.11$ & 0.818 & $0.89 \pm 0.09$ & 0.986 \\
\hline \multicolumn{7}{|l|}{ Ventral } \\
\hline 1 & $0.15 \pm 0.02$ & 0.161 & $0.37 \pm 0.07$ & 0.41 & $0.37 \pm 0.02$ & 0.414 \\
\hline 2 & $0.19 \pm 0.02$ & 0.21 & $0.47 \pm 0.02$ & 0.52 & $0.56 \pm 0.02$ & 0.618 \\
\hline
\end{tabular}

Force per unit area: $1.11 \mathrm{mN} / \mathrm{mm}^{2}$, derived from Landmesser and Morris (1975)

Table 2

Average length of tissue $(l)$, average area separating tibiotarsus and fibula $(A)$ and stiffness $(k)$ of springs, used to represent intervening tissue at $\mathrm{HH} 30, \mathrm{HH} 32$ and HH34. Young's modulus of cartilage (E),

\begin{tabular}{llll}
\hline Stage & $\begin{array}{l}\text { Average length } \\
\text { of tissue }(l)\end{array}$ & $\begin{array}{l}\text { Average area of } \\
\text { tissue }(A)\left(\mathrm{mm}^{2}\right)\end{array}$ & $\begin{array}{l}\text { Stiffness } \\
\left(k=E^{*} A / l\right)(\mathrm{kN} / \mathrm{mm})\end{array}$ \\
\hline HH30 & 0.5 & 0.30 & 0.3 \\
HH32 & 0.9 & 1.9 & 1.1 \\
HH34 & 1.1 & 2.2 & 1.2 \\
\hline
\end{tabular}

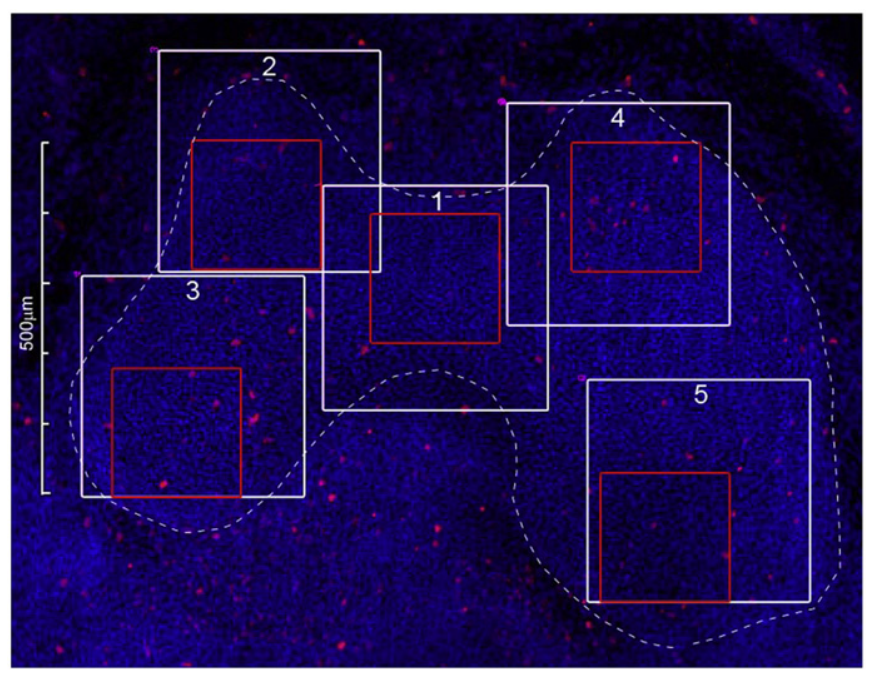

Fig. 2. Transverse sections through the distal femur (outlined in white) were immunostained for Phospho-histone H3 (red) and DAPI stained (blue), were subdivided into five separate regions (1-5) corresponding to the region adjacent to the intercondylar fossa ( 1$)$, the dorsal and ventral side of the medial condyle $(2,3)$ and the dorsal and ventral side of the lateral condyle $(4,5)$ and examined by confocal microscopy. Within each region cell densities were calculated from two independent sections per specimen and 3 specimens per stage, within a region of $1.44 \mathrm{~mm}^{2}$, indicated by red boxes. (For interpretation of the references to color in this figure legend, the reader is referred to the web version of this article.)

markers to be used in selecting consistent section planes. Outlines, capturing local shape changes in the distal femur, were overlaid so that the medial and lateral sides of the femora in the sections were parallel and the midlines of the intercondylar fossae were overlapping.

\subsubsection{Determining rates of cell proliferation}

The proportion of proliferating cells was determined using the mitosis marker anti-phospho-histone $\mathrm{H} 3$ PABs (Millipore) and the secondary antibody Cy3 goat anti-rabbit IGg (Jackson immuno) on 25-30 $\mu \mathrm{m}$ longitudinal cryo-sections, mounted in ProLong Gold anti-fade reagent with DAPI (Invitrogen).

The density of proliferating chondrocytes was determined in five cartilage regions corresponding to the intercondylar fossa, and the dorsal and ventral portions of the medial and lateral condyles, imaged separately (Fig. 2(1-5)) using an Olympus FV1000 point scanning confocal microscope. The numbers of chondrocytes and proliferating cells were counted within a box $1.44 \mathrm{~mm}^{2}$ (Fig. 2(1-5), red box) for two independent focal planes on two sections per specimen $(n=3)$. Results were analysed using $R$.

\section{Results}

\subsection{Biophysical simulation of the distal femur during development}

FE analysis predicted the biophysical stimuli throughout the developing knee joint. Focusing on the distal femur, a prominent feature of the pattern was a peak in stimuli located adjacent to the intercondylar fossa (Fig. 3, arrowhead in A). This peak was present in both patterns of the Von Mises stress and maximum principal stress in both mid-flexion and mid-extension (Figs. 3A, B and 4C, D) as well as patterns of maximum principal strain, fluid velocity and hydrostatic pressure (not shown). Of the stimuli analysed here patterns of minimum principal stress varied the most between the flexion and extension loading steps (Figs. $3 \mathrm{C}$ and $4 \mathrm{E}, \mathrm{F}$ ). In the first step the magnitude of stimuli varied little between the five locations examined (Fig. 4E). A peak of compression adjacent to the intercondylar fossa appears in the second loading step (Fig. 3C, iv).

Within the femoral condyles the predicted loading was consistently higher on the dorsal surface compared to the ventral surfaces in duplicate models. In the flexion phase the stimuli within the lateral and medial condyle appeared to be largely equivalent (Fig. 4C, E). However, in the extension step the magnitude of loading on the medial side of the knee was higher (Fig. 4D, F). The same pattern was predicted for both maximum and minimum principal strains and fluid flow across all three stages.

\subsection{Biophysical stimuli in emerging tissues of the interzone}

Focusing on the patterns of biophysical stimuli in the emerging tissues of the interzone, stress and strain were found to be similar, so only patterns of the Von Mises stress are shown, together with fluid velocity and hydrostatic pressure (Fig. 5). The region of the future patella, which develops from the capsular condensation and the interzone, appeared to experience significantly greater stress, fluid velocity and pore pressure than the rest of the interzone across the three stages (Fig. 5C-E indicated by ${ }^{*}$ ). The stress on the emerging patella was predicted to be approximately four times that predicted for the future articular cartilage and cavity (Fig. 5C). The chondrogenous layers, which first emerge at HH32 (Roddy et al., 2009), develop in a region characterised by an elevated fluid velocity (Fig. 5D, red arrows) and a region of elevated pore pressure midway between the two cartilage anlaga (Fig. 5E, blue arrows), approximately where the cavity should form. Cavity formation begins at approximately HH34 (Roddy et al., 2009). It was also noted that the trend of elevated stimuli 


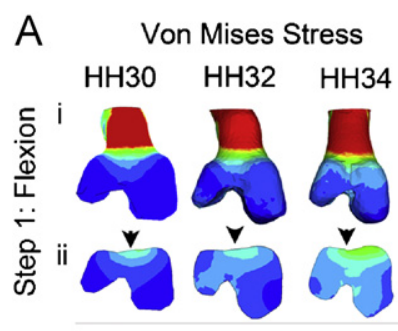

\begin{tabular}{|lll}
$B$ & Max Prin. Stresss \\
$\mathrm{HH} 30$ & $\mathrm{HH} 32$ & $\mathrm{HH} 34$
\end{tabular} \mid
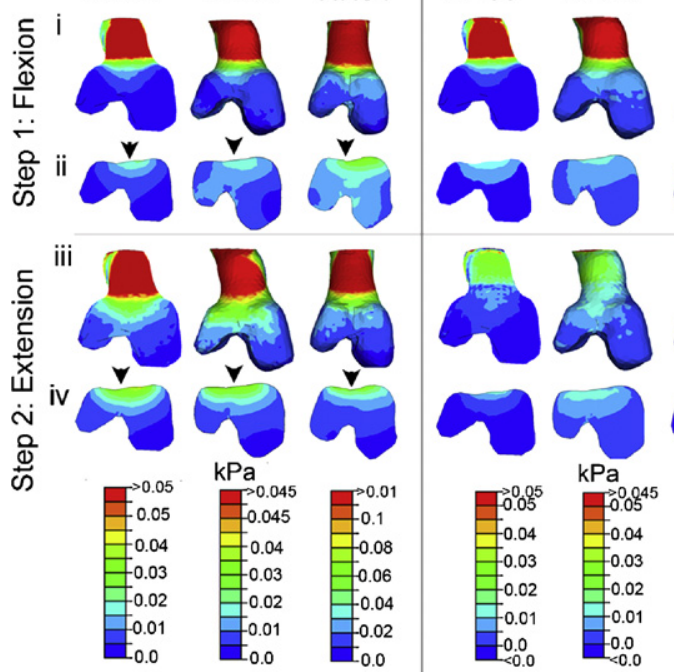
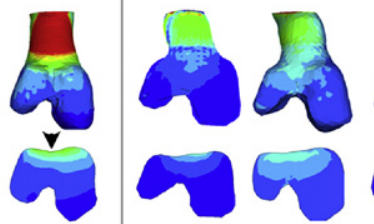

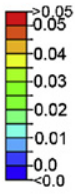
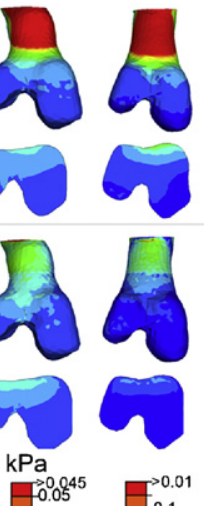

$F_{0.04}^{0.05}$

$\because 0.04$

0.03

$E_{0.01}^{0.02}$

$=0.01$

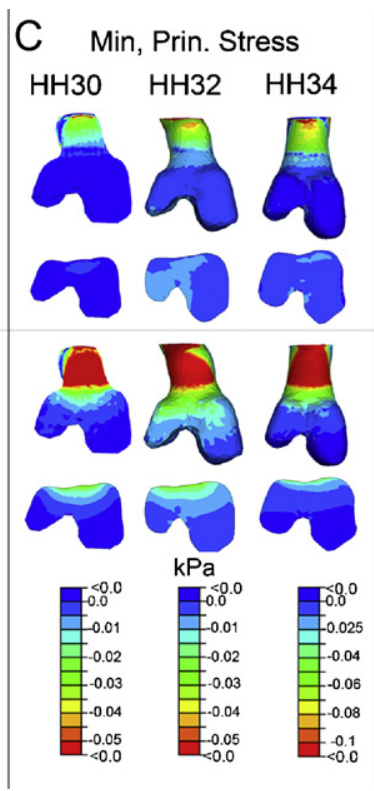

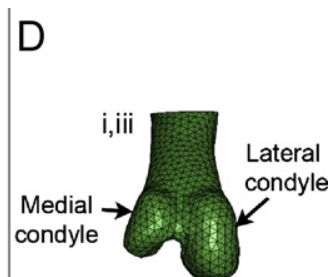
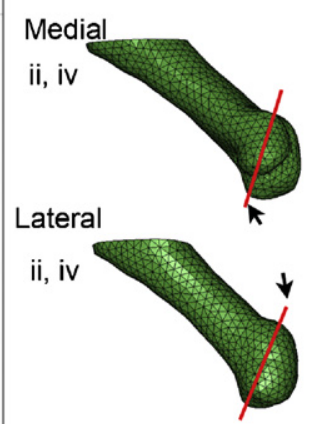

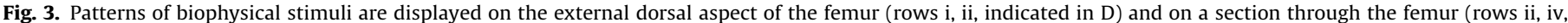

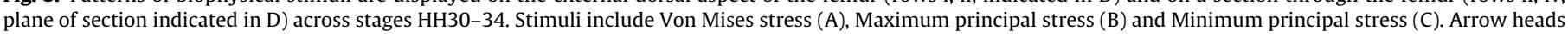
in panel $\mathrm{A}$ indicate the location of the region adjacent to the intercondylar fossa in all the sections.

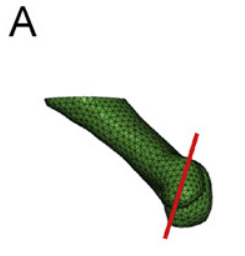

B
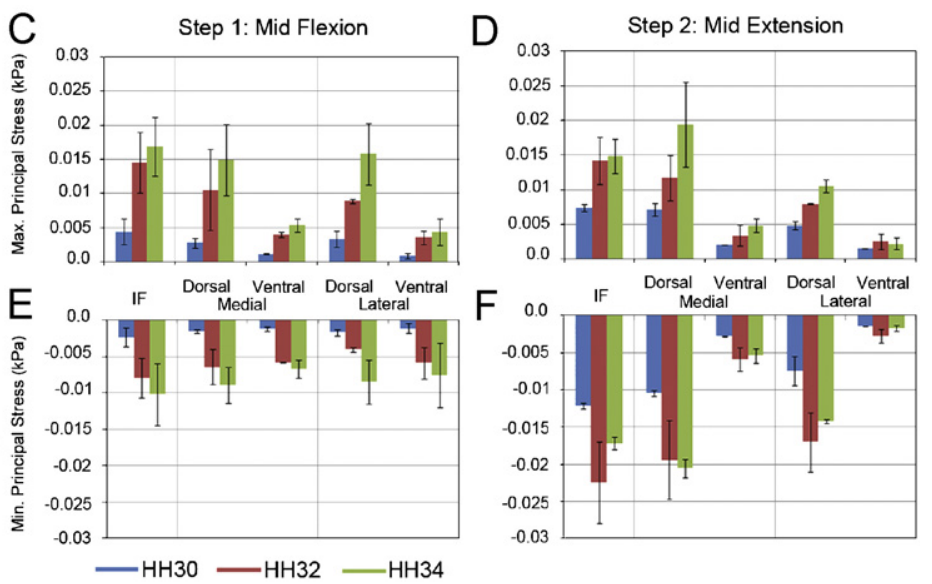

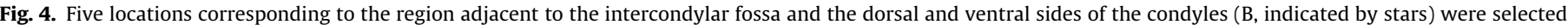

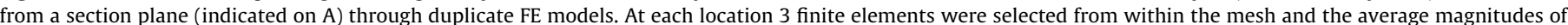

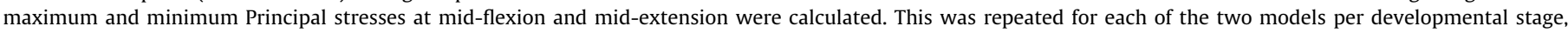

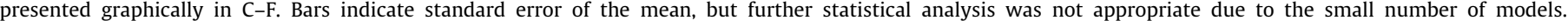
IF; intercondylar fossa.

within the medial side of the femur was also reflected in elevated stress and strain in the medial aspect of the interzone (not shown).

\subsection{Growth of the femoral condyles between HH3O and HH34}

Growth of the femoral condyles was assessed by comparing outlines of cartilage tissue from virtual sections of OPT representations at stages $\mathrm{HH} 30, \mathrm{HH} 32$ and HH34 (Fig. 6a). Relative growth of the condyles was compared by dividing each section in two down the midline of the intercondylar fossa and calculating the area of each side (Fig. 6b). This was repeated for four specimens per stage. At $\mathrm{HH} 30$, the distal femur was a relatively simple structure featuring a larger lateral condyle (Fig. 6c); the cross-sectional area of the medial condyle was $44.6 \pm 3 \%$ of the lateral condyle in this plane. At HH32, the medial side had grown to $51.1 \pm 5 \%$ of the lateral side, an increase of approximately $6.5 \%$ with respect to HH30. The most dramatic growth occurred between stages HH32 and HH34 (Fig. 6f) with the emergence of a more complex shape including several protrusions on the ventral aspect of the lateral condyle (Fig. 6g). The area of the medial condyle was now $65.4 \pm 8 \%$ of the lateral condyle, an increase of approximately $14.3 \%$ on stage HH32 and $20.8 \%$ on stage $\mathrm{HH} 30$. Therefore the medial condyle grows at an accelerated rate relative to the lateral condyle.

\subsection{Proliferation, growth and the emergence of shape}

Analysis of the relative number of proliferating cells in 5 locations across the distal femur; the dorsal and ventral aspects of the medial and lateral condyles and the intercondylar fossa (exact locations outlined in Fig. 2) revealed a dramatic increase at HH32 relative to $\mathrm{HH} 30$ (s.e. $=0.1, p<0.01$ ) and $\mathrm{HH} 34$ (s.e. $=0.1, p<0.01$ ) (Fig. 7). Comparing individual regions it became apparent that the 

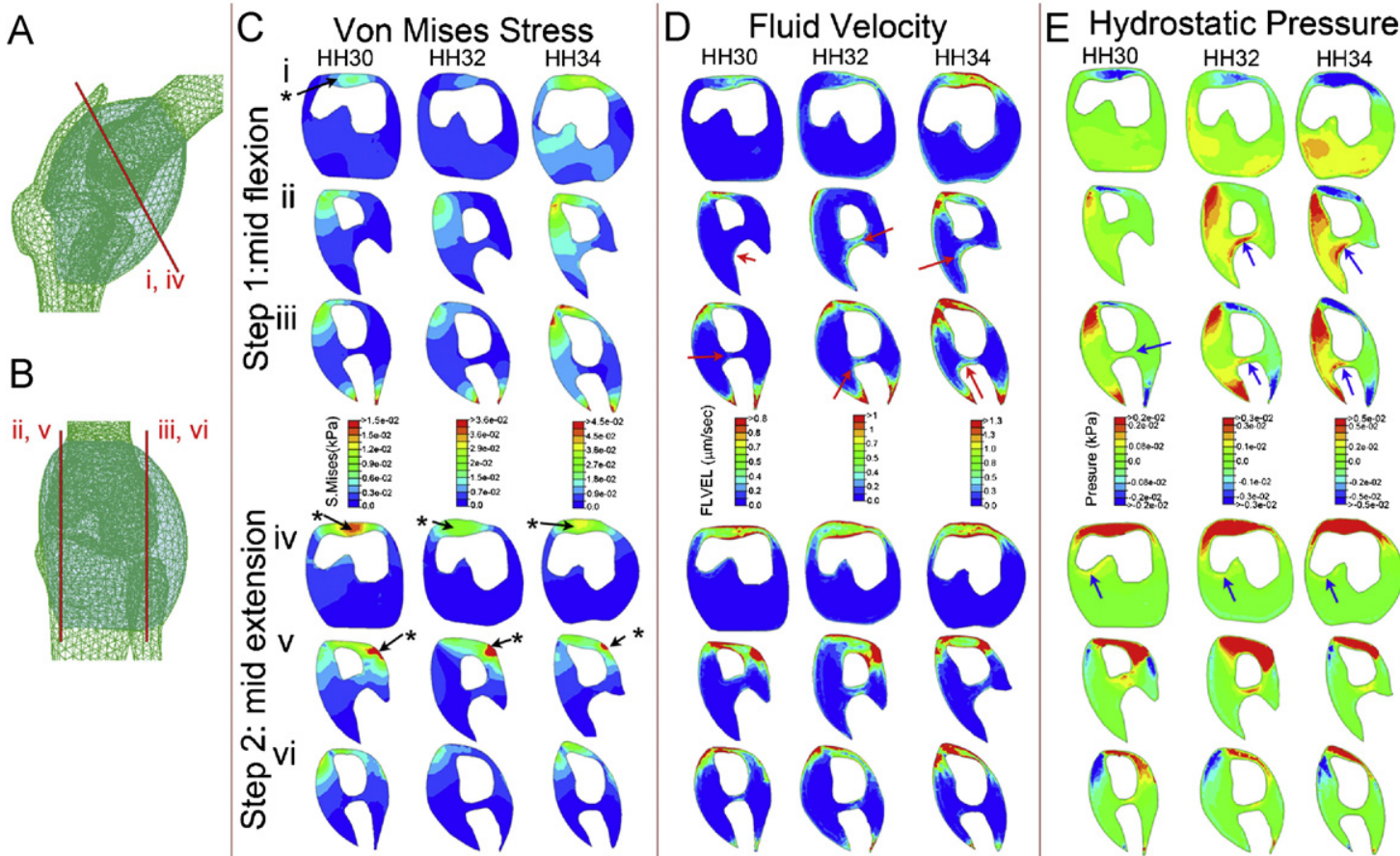

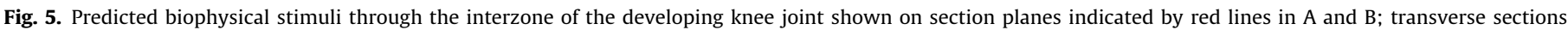

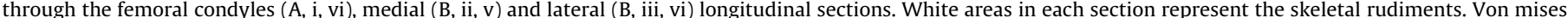

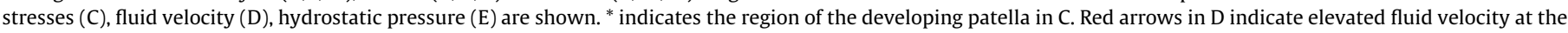

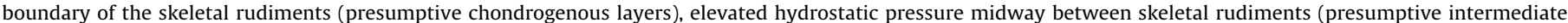
layer) is indicated by blue arrows. (For interpretation of the references to color in this figure legend, the reader is referred to the web version of this article.)

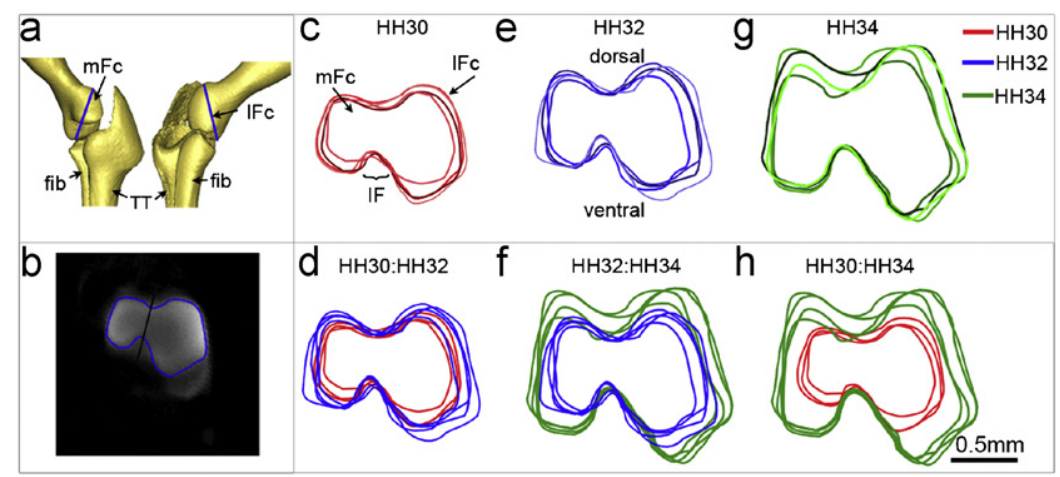

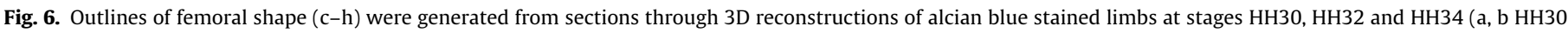

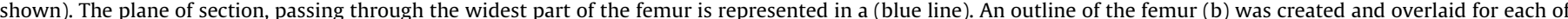

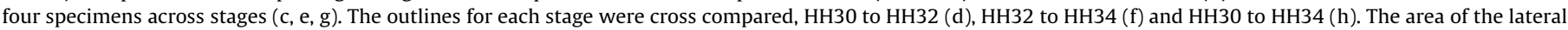

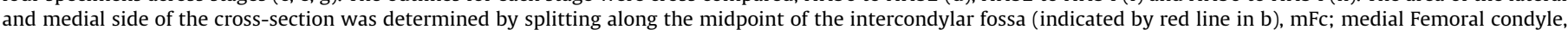

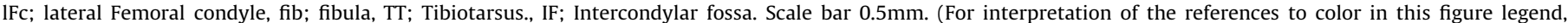
the reader is referred to the web version of this article.)

number of proliferating cells varied depending on location within the femur. At HH32, the increase in proliferating cells on the medial side of the knee was more pronounced, with a significantly greater number of proliferating cells on the medial side at $\mathrm{HH} 32$ (s.e. $=0.1, p=0.01)$. This was investigated using a generalised linear mixed effects model where multiple sections were nested within individuals $(R)$ in order to take account of the nested nature of the data and verified using a sequential Bonferroni test. Proliferation at HH34 was lower than that recorded at HH32. At this stage proliferation still appeared elevated on the medial side relative to the lateral side of the femur, although significance was not reached at $\mathrm{HH} 30$ or HH34 (R).

\section{Discussion}

In this paper we hypothesised that both joint shape and cell proliferation co-localise with the pattern of biophysical stimuli generated by embryonic muscle contraction. Anatomically accurate FE models of embryonic knee, across three stages of development, revealed the complex dynamic pattern of biophysical stimuli in the distal femur, including a consistent increase in the loading of the medial condyle. Analysis of the femoral condyles showed that the medial condyle grows more than the lateral during this developmental period. Differential rates of cell proliferation revealed a possible mechanism behind 


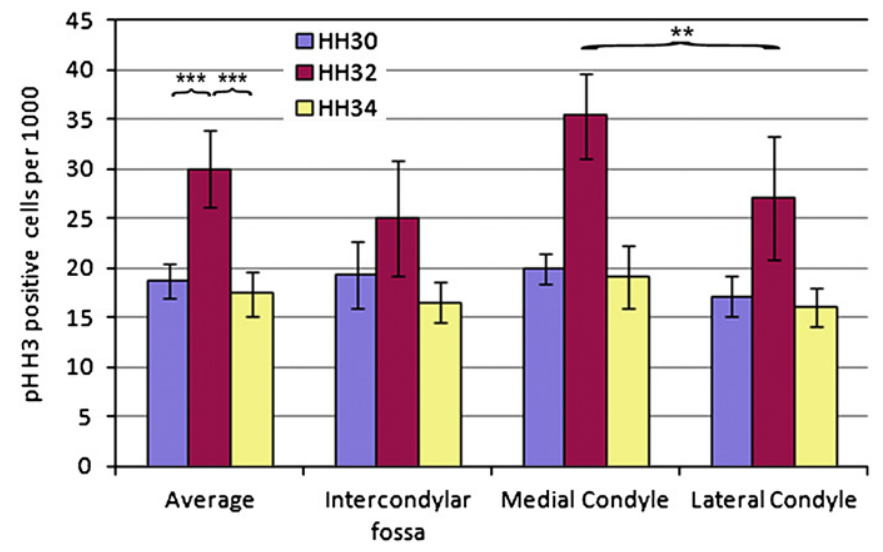

Fig. 7. The number of phospho Histone $\mathrm{H} 3$ positive cells normalized to cell density was determined in selected regions of the intercondylar fossa and medial and lateral condyles (Fig. 2; dorsal and ventral regions within the condyles were combined, average is the combination of all 5) at stages $\mathrm{HH} 30, \mathrm{HH} 32$ and HH34 (2 sections from 3 specimens per stage). Error bars represent standard error. ${ }^{* *}: p<0.01,{ }^{* * *}: p<0.001$.

this growth, whereby the medial condyle appeared to have a higher proportion of proliferating cells than the other regions. This trend was statistically significant at $\mathrm{HH} 32$ but not at $\mathrm{HH} 30$ or $\mathrm{HH} 34$, possibly due to the small sample number $(n=3)$. Thus a relationship was found between elevated cartilage growth and cell proliferation in the medial condyle of the knee; this, in turn, associated with an increase in the magnitude of the biophysical stimuli experienced within this condyle. It was also noted that the proportion of proliferating cells in the region adjacent to the intercondylar fossa remained constant over time and this region, in turn, is highly loaded in both mid-flexion and mid-extension. Thus the consistently high mechanical loading in the region adjacent to the intercondylar fossa is associated with a constant rate of cell proliferation. Therefore the variable pattern of loading experienced by the condyles may have a stimulatory effect on outgrowth, while the consistently elevated loading near the intercondylar fossa maintains a consistent rate of cell proliferation. Growth would then be encouraged by dynamic loading in the condyles, while the higher less dynamic loads near the intercondylar fossa, predicted by the model, maintained a consistent rate of proliferation.

The mechanoregulation of chondrocyte proliferation and biosynthesis has been extensively studied in a varied range of culture systems and stimuli (reviewed in McMahon et al., 2008b). It is difficult to assess the individual effects of each of these potential stimuli due to the differences between experiments; however some trends do emerge from the data. Continuous loads or high frequency, high magnitude loads inhibits cell processes such as matrix synthesis and growth (Buschmann et al., 1995; Davisson et al., 2002; Fukuda et al., 1997; Sironen et al., 2002; Wu and Chen, 2000), while low magnitude dynamic loading stimulates matrix synthesis (Buschmann et al., 1995; Davisson et al., 2002; Fukuda et al., 1997; Wu and Chen, 2000). Reduced chondrocyte proliferation was also recorded in DMB immobilised chick growth plates (Germiller and Goldstein, 1997). Mechanical forces have also been shown to regulate cell differentiation; particularly adult MSCs have demonstrated an ability to differentiate into chondrocytes in response to intermittent hydrostatic pressure (Angele et al., 2003), and cyclic compressive loading (Angele et al., 2003; Huang et al., 2004) and cyclic strain (McMahon et al., 2008a).

Some simplifications included in the models should be mentioned. The choice to focus only on the loads applied to the knee by the thigh musculature overlooks what influence the shank muscles may play in the emergence of shape. However, while it is likely that the shank muscles exert forces on the knee joint, such forces would be predominately applied to the lower portions of the tibiotarsus, as represented in Nowlan et al. (2008a). Since this work focused on the patterns of loading rather than the magnitude of the individual loads, a single measurement for muscle force (derived from Landmesser and Morris, 1975) was deemed to be sufficient, particularly as muscle size is already accounted for. Another limitation is the shape of the mesh model representing the interzone, particularly its ventral side, which was defined only by adjacent muscles masses. However patterns of stimuli at or close to the ventral boundary are not interpreted here, with focus on the deeper tissues of the interzone and regions under the capsule.

The material properties of both embryonic cartilage and interzone tissues were measured using AFM and analysed using the Hertz model. A proportion (20-40\%) of the force curves were removed during analysis because the height variation across the section being scanned was larger than the piezo range, rendering the curves unusable. Other curves did not fit the Hertz model (approximately 20\%) and had to be eliminated from the analysis. The Hertz model makes a number of assumptions including that the material being tested is both homogenous and isotropic. Failure to meet these conditions due to local changes in the extracellular matrix or cell density could explain why certain curves do not always fit the Hertz model. The cartilage material properties $(0.535 \pm 0.072 \mathrm{kPa})$ measured by AFM were softer than those previously published for other cartilage tissues using different methods and older cartilage. Tanck et al. (2004) derived a Young's modulus of $1.11 \pm 0.62 \mathrm{MPa}$, while Mikic et al. (2004) recorded $0.08 \mathrm{MPa}$. However our results were in the range of values previously recorded for cartilage extracellular matrix determined using AFM (Allen and Mao, 2004). For the purposes of the FE model, the relative stiffness of the cartilage and the interzone $(0.535 \pm 0.072$ and $0.287 \pm 0.066 \mathrm{kPa}$, respectively) was of particular importance providing important information on the relative stiffness of embryonic tissues for the first time. It would be expected that the material properties of embryonic cartilage and the interzone would change over time. We did not include this in the model because of the technical difficulties and time constraints associated with using AFM to derive material properties.

A limited number of studies have aimed to investigate the mechanobiology of shape emergence in joints (Heegaard et al., 1999; Shefelbine and Carter, 2004; Shefelbine et al., 2002). While these models cannot be compared directly to the 3D model presented in this paper, they do support the role of mechanoregulation in joint morphogenesis. Nowlan et al. (2008a) created an FE model based on realistic tibiotarsal morphologies, revealing the importance of dynamic patterns of biophysical stimuli in bone development in the embryo. This paper indicates a regulatory link between cell proliferation and tissue growth in the embryo with the pattern of biophysical stimuli generated by muscle contraction; however further analysis of how these patterns influence cell behaviour is required.

Previous studies of joint development in the chick have indicated that preventing muscle contraction can prevent articular cartilage and patella formation (Drachman and Sokoloff, 1966; Mikic et al., 2000). FE analysis of the interzone indicated that the articular cartilages and patella develop under the influence of very specific patterns of biophysical stimuli. The chondrogenous layers, which ultimately form the articular cartilages (Mitrovic, 1977), emerge from locations, which experience a very distinct pattern of elevated fluid flow of between 0.4 and $0.8 \mu \mathrm{m} / \mathrm{s}$ depending on the stage. In contrast the patella develops under much higher magnitudes of stress, fluid velocity and pore pressure. Prendergast et al. (1997) proposed that a combination of shear strain and fluid flow regulates cell differentiation in the skeleton, in a model 
corroborated by experimental results (Isaksson et al., 2006; Khayyeri et al., 2009). The boundaries for chondrogenic differentiation were also corroborated by analysing the strain and fluid experienced by MSCs undergoing chondrogenesis in a 3D scaffold (McMahon et al., 2008a). The biophysical stimuli to which the developing articular cartilage are exposed are well within the range proposed by Prendergast et al. (1997). The specific patterns of biophysical stimulation in the joint interzone could indicate that mechanoregulation is vital not only for the regulation of shape in the embryonic skeleton, but also vital for the definition of cell fate and resulting tissue boundaries.

\section{Conflict of interest statement}

The authors (K.A. Roddy, G.M. Kelly, M.H. van Es, P. Murphy and P.J. Prendergast) declare that they have no conflict of interest. We confirm that all authors were fully involved in the study and preparation of this manuscript, and that the material within has not been submitted for publication elsewhere.

\section{Acknowledgements}

This work was partially funded by a studentship from Trinity College Dublin (SFI AOIP interdisciplinary programme) and by Science Foundation Ireland (Programme Award 02/IN1/B267 and 07/IN1/B931). The authors thank Dr. Jason Kilpatrick and Dr. Niamh Nowlan for their contribution to this work.

\section{References}

Allen, D.M., Mao, J.J., 2004. Heterogeneous nanostructural and nanoelastic properties of pericellular and interterritorial matrices of chondrocytes by atomic force microscopy. Journal of Structural Biology 145, 196-204.

Angele, P., Yoo, J.U., Smith, C., Mansour, J., Jepsen, K.J., Nerlich, M., Johnstone, B. 2003. Cyclic hydrostatic pressure enhances the chondrogenic phenotype of human mesenchymal progenitor cells differentiated in vitro. Journal of Orthopaedic Research 21, 451-457.

Buschmann, M., Gluzband, Y., Grodzinsky, A., Hunziker, E., 1995. Mechanical compression modulates matrix biosynthesis in chondrocyte/agarose culture. Journal of Cell Science 108, 1497-1508.

Carter, D.R., Wong, M., 1988. The role of mechanical loading histories in the development of diarthrodial joints. Journal of Orthopaedic Research 6 804-816

Davisson, T., Kunig, S., Chen, A., Sah, R., Ratcliffe, A., 2002. Static and dynamic compression modulate matrix metabolism in tissue engineered cartilage. Journal of Orthopaedic Research 20, 842-848.

Drachman, D.B., Sokoloff, L., 1966. The role of movement in embryonic joint development. Developmental Biology 14, 401-420.

Fukuda, K., Asada, S., Kumano, F., Saitoh, M., Otani, K., Tanaka, S., 1997. Cyclic tensile stretch on bovine articular chondrocytes inhibits protein kinase $C$ activity. Journal of Laboratory and Clinical Medicine 130, 209-215.

Germiller, J.A., Goldstein, S.A., 1997. Structure and function of embryonic growth plate in the absence of functioning skeletal muscle. Journal of Orthopaedic Research 15, 362-370.

Hamburger, V., Hamilton, H.L., 1951. A series of normal stages in the development of the chick embryo. Journal of Morphology 88, 49-92.

Heegaard, J.H., Beaupré, G.S., Carter, D.R., 1999. Mechanically modulated cartilage growth may regulate joint surface morphogenesis. Journal of Orthopaedic Research 17, 509-517.

Hertz, H., 1882. Ueber die Berührung fester elastischer Körper. Journal für die Reine und Angewandte Mathematik. 1882, 156-171.

Huang, C.Y.C., Kristen, L.H., Lauren, E.F., Yubo, S., Herman, S.C., 2004. Effects of cyclic compressive loading on chondrogenesis of rabbit bone-marrow derived mesenchymal stem cells. Stem Cells 22, 313-323.

Hutter, J.L., Bechhoefer, J., 1993. Calibration of atomic-force microscope tips. Review of Scientific Instruments 64, 1868-1873.

Isaksson, H., Wilson, W., van Donkelaar, C.C., Huiskes, R., Ito, K., 2006. Comparison of biophysical stimuli for mechano-regulation of tissue differentiation during fracture healing. Journal of Biomechanics 39, 1507-1516.

Kelly, D.J., Prendergast, P.J., 2005. Mechano-regulation of stem cell differentiation and tissue regeneration in osteochondral defects. Journal of Biomechanics 38 1413-1422.

Khayyeri, H., Checa, S., Tägil, M., Prendergast, P.J., 2009. Corroboration of mechanobiological simulations of tissue differentiation in an in vivo bone chamber using a lattice-modeling approach. Journal of Orthopaedic Research 27, 1659-1666

Koyama, E., Shibukawa, Y., Nagayama, M., Sugito, H., Young, B., Yuasa, T., Okabe, T., Ochiai, T., Kamiya, N., Rountree, R.B., Kingsley, D.M., Iwamoto, M., EnomotoIwamoto, M., Pacifici, M., 2008. A distinct cohort of progenitor cells participates in synovial joint and articular cartilage formation during mouse limb skeletogenesis. Developmental Biology 316, 62-73.

Landmesser, L., Morris, D.G., 1975. The development of functional innervation in the hind limb of the chick embryo. The Journal of Physiology 249, 301-326.

McMahon, L., Reid, A., Campbell, V., Prendergast, P., 2008a. Regulatory effects of mechanical strain on the chondrogenic differentiation of MSCs in a collagen-GAG Scaffold: experimental and computational analysis. Annals of Biomedical Engineering 36, 185-194.

McMahon, L.A., O’Brien, F.J., Prendergast, P.J., 2008b. Biomechanics and mechanobiology in osteochondral tissues. Regenerative Medicine 3, 743-759.

Mikic, B., Isenstein, A.L., Chhabra, A., 2004. Mechanical modulation of cartilage structure and function during embryogenesis in the chick. Annals of Biomedical Engineering 32, 18-25

Mikic, B., Johnson, T.L., Chhabra, A.B., Schalet, B.J., Wong, M., Hunziker, E.B., 2000. Differential effects of embryonic immobilization on the development of fibrocartilaginous skeletal elements. Journal of Rehabilatation Research and Development 37, 127-133.

Mitrovic, D.R., 1977. Development of the metatarsophalangeal joint of the chick embryo: morphological, ultrastructural and histochemical studies. American Journal of Anatomy 150, 333-347.

Murray, P.D., Drachman, D.B., 1969. The role of movement in the development of joints and related structures: the head and neck in the chick embryo. Journal of Embryology and Experimental Morphology 22, 349-371.

Nowlan, N.C., Murphy, P., Prendergast, P.J., 2008a. A dynamic pattern of mechanical stimulation promotes ossification in avian embryonic long bones. Journal of Biomechanics 41, 249-258.

Nowlan, N.C., Prendergast, P.J., Murphy, P., 2008b. Identification of mechanosensitive genes during embryonic bone formation. PLoS Computational Biology 4 e1000250.

Osborne, A.C., Lamb, K.J., Lewthwaite, J.C., Dowthwaite, G.P., Pitsillides, A.A., 2002. Short-term rigid and flaccid paralyses diminish growth of embryonic chick limbs and abrogate joint cavity formation but differentially preserve pre-cavitated joints. Journal of Musculoskeletal and Neuronal Interaction 2, 448-456.

Persson, M., 1983. The role of movements in the development of sutural and diarthrodial joints tested by long-term paralysis of chick embryos. Journal of Anatomy 137 (Pt 3), 591-599.

Prendergast, P.J., Huiskes, R., Søballe, K., 1997. Biophysical stimuli on cells during tissue differentiation at implant interfaces. Journal of Biomechanics 30, 539-548.

Radhakrishnan, P., Lewis, N.T., Mao, J.J., 2004. Zone-specific micromechanical properties of the extracellular matrices of growth plate cartilage. Annals of Biomedical Engineering 32, 284-291.

Roddy, K.A., Nowlan, N.C., Prendergast, P.J., Murphy, P., 2009. 3D representation of the developing chick knee joint: a novel approach integrating multiple components. Journal of Anatomy 214, 374-387.

Rotsch, C., Braet, F., Wisse, E., Radmacher, M., 1997. AFM imaging and elasticity measurements on living rat liver macrophages. Cell Biology International 21, 685-696.

Shefelbine, S.J., Carter, D.R., 2004. Mechanobiological predictions of growth front morphology in developmental hip dysplasia. Journal of Orthopaedic Research 22, 346-352.

Shefelbine, S.J., Tardieu, C., Carter, D.R., 2002. Development of the femoral bicondylar angle in hominid bipedalism. Bone 30, 765-770.

Sironen, R.K., Karjalainen, H.M., Elo, M.A., Kaarniranta, K., Torronen, K., Takigawa, M., Helminen, H.J., Lammi, M.J., 2002. cDNA array reveals mechanosensitive genes in chondrocytic cells under hydrostatic pressure. Biochimica et Biophysica Acta 1591, 45-54.

Sneddon, I.N., 1965. The relation between load and penetration in the axisymmetric Boussinesq problem for a punch of arbitrary profile. International Journal of Engineering Science 3, 47-57.

Stevens, S.S., Beaupré, G.S., Carter, D.R., 1999. Computer model of endochondra growth and ossification in long bones: biological and mechanobiological influences. Journal of Orthopaedic Research 17, 646-653.

Stolz, M., Raiteri, R., Daniels, A.U., Van Landingham, M.R., Baschong, W., Aebi, U., 2004. Dynamic elastic modulus of porcine articular cartilage determined at two different levels of tissue organization by indentation-type atomic force microscopy. Biophysical Journal 86, 3269-3283.

Summerhurst, K., Stark, M., Sharpe, J., Davidson, D., Murphy, P., 2008. 3D representation of Wnt and Frizzled gene expression patterns in the mouse embryo at embryonic day 11.5 (Ts19). Gene Expression Patterns 8, 331-348.

Tanck, E., Blankevoort, L., Haaijman, A., Burger, E.H., Huiskes, R., 2000. Influence of muscular activity on local mineralization patterns in metatarsals of the embryonic mouse. Journal of Orthopaedic Research 18, 613-619.

Tanck, E., Van Donkelaar, C.C., Jepsen, K.J., Goldstein, S.A., Weinans, H., Burger, E.H., Huiskes, R, 2004. The mechanical consequences of mineralization in embryonic bone. Bone 35, 186-190.

Wilsman, N.J., Leiferman, E.M., Fry, M., Farnum, C.E., Barreto, C., 1996. Differential growth by growth plates as a function of multiple parameters of chondrocytic kinetics. Journal of Orthopaedic Research 14, 927-936.

$\mathrm{Wu}$, Q.Q., Chen, Q., 2000. Mechanoregulation of chondrocyte proliferation, maturation, and hypertrophy: ion-channel dependent transduction of matrix deformation signals. Experimental Cell Research 256, 383-391. 